\title{
Convective mixing in intermediate mass stars
}

\author{
Alessandro Bressan \\ Scuola Internazionale Superiore di Studi Avanzati - via Bonomea, 265 - 34136, Trieste, IT \\ email: sbressan@sissa.it
}

\begin{abstract}
Stellar evolution can nowadays be modelled with a high degree of accuracy and completeness up to the most advanced stages. However in spite of the progresses, complex physical processes exist that still suffer of large uncertainties even in the most placid evolutionary phases. The straightforward drawback is that models lose their predictive power and this is particularly critical for stellar population synthesis. Here I will focus on one of such processes, convective mixing, and briefly review potentially helpful observational tests to decipher its efficiency during the main nuclear burning phases of intermediate mass stars.
\end{abstract}

Keywords. convection, stars: interiors, stars: evolution, stars: AGB and post-AGB

\section{Convective mixing during the H-Burning phase}

The evolution of intermediate mass stars during and after the main sequence is particularly sensitive to the efficiency of mixing processes originating in the convective core. While we know that convection is certainly the main mixing agent, very little is known about its extent into the surrounding stable layers. Constraints on the "overshooting" scale above the unstable region, of $\sim 0.25$ pressure scale heights $\left(\mathrm{H}_{P}\right)$, have been derived from comparison with turn-off stars in Open Clusters (see Bressan et al. 2012, Girardi et al. 2009) and in binary systems (Torres et al. 2014), from asteroseismology of main sequence stars (Silva Aguirre et al. 2013), and from the morphology of the Red Clumps in star clusters (Girardi et al. 2013). Note that rotational mixing may lead to very similar observational effects (Brandt \& Huang 2015), rendering the above calibration quite illusory.

\section{Convective mixing during the He-burning phase}

Ranked by their efficiency, four main mixing schemes have been proposed for Heburning stars (Castellani et al. 1971, Bressan et al. 1986): Bare Schwarzschild -BS-, Local Overshooting -LO-, Semi-convection -SC-and, finally, Non Local Overshooting -NLOthat, contrary to the previous ones, contains a free parameter. At increasing mixing efficiency the central He-burning lifetime increases. The subsequent Early Asymptotic Giant Branch (EAGB) phase records the integrated effects of He-core mixing, being shorter and more luminous for a larger efficiency. The $R$ 2 ratio, between the observed number of stars in the EAGB and in the Horizontal Branch in globular clusters, is a classical powerful diagnostic for the efficiency of mixing (Buonanno et al.1985), but it cannot clearly discriminate between e.g. $L O+S C$ and $N L O$. Bossini et al. (2015) argue that the combination of the luminosity of the EAGB bump (classical) and the He-burning period spacing of gravity modes (seismic) tests, provides a decisive diagnostic for the efficiency of mixing during central He-burning. Preliminary comparisons with seismic constraints obtained from Kepler light curves of the APOKASC red giant sample (Pinsonneault et al. 2014, Mosser et al. 2014) favor a model with a moderate/high adiabatically stratified overshooting layer of $\sim 0.5 H_{p}$ above the unstable region (Bossini et al. (2015)). 


\section{Overshooting at the bottom of the convective envelope}

Moderate overshooting $\left(0.25-0.6 H_{P}\right)$ at the bottom of the convective envelope is invoked to solve the discrepancy between the observed and modelled location of the red giant branch bump (Alongi et al. 1991) and as an alternative way to solve the Li problem (Fu et al.2015). It can also help to better reproduce solar oscillations data (ChristensenDalsgaard et al. 2011). Its main effect on intermediate mass stars is that of favoring the extension of blue loops (Alongi et al. 1991). The most recent simulations of blue loops in the observed CM diagrams of WLM, NGC 6822, Sextans A and Sagittarius dwarf irregular galaxies, performed with the observed spectroscopic metallicities which is a necessary condition to avoid degeneracy, require an overshooting of $\geqslant 2 \mathrm{H}_{P}$ below the bottom of the convective envelope, in the previous red giant phases (Tang et al.2014, 2015). Similar values are needed to enhance the efficiency of the carbon dredge up during the AGB phase (Kamath et al. 2012) and are also indicated by recent 3D simulations (e.g. Figure 4 in Viallet et al.2013).

\section{Conclusions}

The efficiency of mixing beyond the formal unstable regions is one of the most uncertain factors in stellar astrophysics, affecting the lifetimes of H-burning and following evolutionary phases (HB, AGB), and their luminosities and effective temperatures (main sequence termination, clump He-stars, blue He burning stars). The indications arising from the comparison with observations of intermediate mass stars in different evolutionary phases are somehow conflicting, suggesting that we are still far from a physically sounded theory for stellar evolution. Asteroseismology and new 3D modelling (e.g. Arnett et al. 2015) will likely provide a viable solution in the next few years.

\section{Acknowledgements}

A. Bressan acknowledges support from ERC project STARKEY, G.A. n. 615604.

\section{References}

Alongi, M., Bertelli, G., Bressan, A., \& Chiosi, C. 1991, A\&A, 244, 95

Arnett et al. 2015, ApJ, 809, 30

Bossini, D. et al. 2015, MNRAS, 453, 2290

Brandt, T. D. \& Huang, C. X. 2015 ApJ, 807, 25

Bressan, A., Marigo, P., Girardi, L., et al. 2012 MNRAS, 427, 127

Bressan, A. et al. 1986, Mem. Soc. Astron. Italiana, 57, 411

Buonanno, R., Corsi, C. E., \& Fusi Pecci, F. 1985, A\& A, 145, 97

Castellani, V. et al. 1971, ApESSS, 10, 340

Christensen-Dalsgaard, J. et al. 2011, MNRAS, 414, 1158

Fu, X, Bressan, A., Molaro, P., \& Marigo, P. 2015, MNRAS, 452, 3256

Girardi, L., Rubele, S., \& Kerber, L. 2009 MNRAS, 394, L74

Girardi, L., Marigo, P., Bressan, A., \& Rosenfield. P. 2013 ApJ, 777, 142

Kamath, D., Karakas, A. I., \& Wood, P. R. 2012, ApJ, 746, 20

Mosser, B. et al. 2014, A\&A A, 572, L5

Pinsonneault, M. et al. 2014, ApJS, 215, 19

Silva Aguirre, V., Basu, S., Brandão, I. M. , et al. 2013 ApJ, 769, 141

Tang, et al. 2014, MNRAS, 445, 4287

Tang, et al. 2015, MNRAS, in press.

Torres, G., Vaz, L. P. R., Sandberg, L., Claud, H., \& Claret, A. 2014 AJ, 147, 36

Viallet et al. 2013 ApJ, 769, 1 